\title{
MATHEMATISCHE ANNALEN
}

\author{
BEGRÜNDET 1868 DURCH \\ ALFRED CLEBSCH UND CARL NEUMANN \\ FORTGEFÜHRT DURCH \\ FELIX KLEIN \\ OTTO BLUMENTHAL \\ DAVID HILBERT \\ ERICH HECKE
}

GEGENWÄRTIG HERAUSGEGEBEN VON

\author{
HeinRich BehNKe \\ MÜNSTER (WESTF.) \\ HeINz HopF \\ ZƯRICH
}

KuRt ReIdemeister GÖTTINGEX

\section{BAND}

\author{
RICHARD COURANT \\ NEW YORK \\ GotTrRIED KöтHe \\ IIEIDELBERG
}

Bartel L. van der Waerden ZÜRICH

S PRIN GER - VER LA G

BERLIN · GÖTTINGEN · HEIDELBERG 


\section{Inhalt des 136. Bandes}

(In alphabetischer Ordnung)

Bauer, F.-W., Spezielle Homologiestrukturen . . . . . . . . . . . . . . . . . 348 (Anschrift: Frankfurt/Main, Oederweg 109)

Behnke, H., Otto Blumenthal zum Gedächtnis. . . . . . . . . . . . . . . 387 (Anschrift: Münster/Westf., Rottendorffweg 17)

Bergman, St., A Class of Pseudo-Conformal and Quasi-Pseudo-Conformal Mappings . . . . . . . . . . . . . . . . . . . . . . . . . . . . . . . . . 134 (Anschrift: Applied Mathematics and Statistics Laboratory, Stanford University, Stanford/California USA)

Bremermann, H. J., Die Charakterisierung Rungescher Gebiete durch plurisubharmonische Funktionen . . . . . . . . . . . . . . . . . . . . . . 173 (Anschrift: Department of Mathematics, University of Washington, Seattle 5, Wash. USA)

Cartan, H., Prolongement des espaces analvtiques normaux . . . . . . . . . . 97 (Anschrift: 95 Boulevard Jourdan, Paris XIVe, Frankreich)

Fngel, W., Ganze Cremona-Transformationen von Primzahlgrad in der Ebene. . . 319 (Anschrift: Halle/Saale S 11, Regensburger Str. 20)

Friedman, B., $n$-Commutative Matrices . . . . . . . . . . . . . . . . . . . 343 (Anschrift: Department of Mathematics, University of California, Berkeley 4, California, USA)

Gandy, R. O., Note on a Paper of Kemeny's . . . . . . . . . . . . . . . 466 (Anschrift: The University of Leeds, Math. Dept., Leeds/England)

G ani, J., Elementary Methods for an Occupancy Problem of Storage. . . . . . . 454 (Anschrift: Dept. of Mathematics, University of Western Australia, Nedlands (Western Australia))

Grauert, H., u. R. Remmert. Komplexe Räume. . . . . . . . . . . . . . 245 (Anschrift: Institute for Advanced Study, Princeton, N.Y., USA;

1. Mathematisches Institut der Universität Münster/W., Schloßplatz 2)

Hahn, W., Über die Anwendung der Methode von Ljapunov auf Differenzengleichungen .. . . . . . . . . . . . . . . . . . . . . . 430 (Anschrift: Braunschweig, Maschstr. 3a)

Heins, M., Some Constructive Problems Concerning Analytische Gebilde . . . . . 9 (Anschrift: University of Illinois, Urbana, Illinois, USA)

Hille, E., On Roots and Logarithms of Elements of a Complex Banach Algebra . . 46 (Anschrift: Dept. of Mathematies, Yale University, New Haven, Conn., USA)

Hirzebruch, F., und H. Hopf, Felder von Flächenclementen in 4-dimensionalen Mannigfaltigkeiten. . . . . . . . . . . . . . . . . 156 (Anschrift: Mathematisches Institut der Universität Bonn/Rhein, Wegelerstr. 10; Zollikon/Zürich, Alte Landstr. 37)

Hopf, H.. siehe Hirzebruch, F.

Jacobson, N., Nilpotent Elements in Semi-simple .Jordan Algehras . . . . . . . 375 (Anschrift: Mathematical Department, Yale University, New Haven (Conn.) USA

Kasch, F., und B. Volkmann, Zur Mahlerschen Vermutung über S-Zahlen . . . 442 (Anschrift: Mathematisches Institut der Universität Heidelberg, 'Tiergartenstr.; Mathematisches Institut der Universität Mainz, Saarstr. 21) 
König, H., Eine Charakterisierung der Distributionen endlicher Ordnung . . . . 240 (Anschrift: Aachen/Rhld., An der Schanz 20)

Knobloch, H.-W., u. H. Röhrl, Zum Begriff der analytischen Fortsetzung in algebraischen Funktionenkörpern einer Veränderlichen . . . . . . . . . . . . . 187 (Anschrift: Würzburg, Mathematisches Institut der Universität;

München, Mathematisches Institut der Universität, GeschwisterScholl-Platz 1)

Pinl, M., Minimalflächen fester Gaußscher Krümmung . . . . . . . . . . . . . 34 (Anschrift: Köln-Riehl/Rhein, Stammheimer Straße 34-36)

Remmert, R., siehe Grauert, H.

Rieger, G. J., Einige Sätze über Ideale in algebraischen Zahlkörpern . . . . . . 339 (Anschrift: The University of Maryland, Department of Mathematics, College Park, Md. USA)

Röhrl, H., siehe Knobloch, H.-W.

Schmidt, W., Flächenapproximation beim Jacobialgorithmus. . . . . . . . . . 365 (Anschrift: Montana State University Missoula, Montana, USA)

Sebastião e Silva, J., Les fonctions analytiques comme ultra-distributions dans le calcul opérationnel . . . . . . . . . . . . . . . . . . . . . . . . . . 58 (Anschrift: Lisboa, Portugal, Praça do Arceiro 5, $3^{\circ} \mathrm{D}$ )

Sommer, F., Komplex-analytische Blätterung reeller Mannigfaltigkeiten im $C^{n}$. . 111 (Anschrift: 1. Mathematisches Institut der Universität Münster/Westf., Schloß. platz 2)

Stein, K., Die Existenz komplexer Basen zu holomorphen Abbildungen . . . . . 1 (Anschrift: München 9, Ulmenstraße 14)

Stoll, W., Über meromorphe Abbildungen komplexer Räume I . . . . . . . . . 201 (Anschrift: 41 Einstein Drive, Princeton, N.Y., USA)

Stoll, W., Über meromorphe Abbildungen komplexer Räume II . . . . . . . . . 393 (Anschrift: 41 Einstein Drive, Princeton N.Y., USA)

Sussman, I., A Generalization of Boolean Rings. . . . . . . . . . . . . . . . 326

(Anschrift: Department of Mathematics, University of Santa Clara, Santa Clara. California, USA)

Tietz, H., Zur Realisierung Riemannscher Flächen II . . . . . . . . . . . . . 41 (Anschrift: Mathematisches Institut der Universität, Münster/Westf., Schloßplatz 2)

Tietz, H., Über Teilreihen von Potenzreihen . . . . . . . . . . . . . . . . . 342 (Anschrift: Münster/Westf., Hoyastr. 2a)

Volkmann, B., siehe Kasch, F.

v. d. Waerden, B. L., Zur algebraischen Geometrie 19. Grundpolynom und zuge. ordnete Form . . . . . . . . . . . . . . . . . . . . . . . . . . . . . 139 (Anschrift: Zürich 6, Bionstr. 18)

Walsh, J. L., A Generalization of Faber's Polynomials .

(Anschrift: Harvard University, Nathem. Dept., Cambridge, Mass., USA) 
Kasch, F. und Volkmane, B.

Math. Annalen, Bd. 136, S. 442-453 (1958)

\title{
Zur Mahlerschen Vermutung über S-Zahlen
}

\author{
Von
}

Friedrich Kasch in Heidelberg und Bodo Volkmann in Mainz

1. Bei der Mahlerschen Klasseneinteilung der transzendenten Zahlen betrachtet man bekanntlich $\left.{ }^{1}\right)$, wenn $\mathfrak{P}(n, H)$ die Menge aller Polynome $P(x)$ mit ganzrationalen Koeffizienten von einem Grad $\leqq n$ und einer Höhe $\leqq H$ ist, $\mathrm{zu}$ einer vorgegebenen $\mathrm{Zahl} \xi$ die Größen

$$
w_{n}(H, \xi)=\min _{\substack{P \in \mathcal{Y}(n, H) \\ P(\xi) \neq 0}}|P(\xi)| \quad \text { und } \quad w_{n}(\xi)=\varlimsup_{H \rightarrow \infty} \frac{\log \frac{1}{w_{n}(H, \xi)}}{\log H} .
$$

Setzt man ferner $\vartheta_{n}(\xi)=\frac{w_{n}(\xi)}{n}$, so ergibt sich in der Bezeichnungsweise von [8] der Typ einer (reellen oder komplexen) Zahl $\xi$ als

$$
\vartheta(\xi)=\sup _{n=1}^{\infty} \vartheta_{n}(\xi)
$$

Unter $S$-Zahlen versteht man dann diejenigen, deren Typ positiv und endlich ist. Der kleinste Wert, den $\vartheta(\xi)$ für transzendente Zahlen $\xi$ annehmen kann, ist $\vartheta(\xi)=1$ für reelles und $\vartheta(\xi)=\frac{1}{2}$ für komplexes $\xi$, und eine Vermutung von $\mathrm{K}$. Mahler [7] besagt, daß dies für fast alle reellen bzw. fast alle transzendenten $\xi$ auch wirklich eintritt. Diese Mahlersche Vermutung ist also, da für reelle transzendente $\xi$ auch immer $\vartheta_{n}(\xi) \geqq 1$ ist, in ihrem reellen Teil mit der Aussage äquivalent, daß für fast alle reellen $\xi$ die Gleichungen

$$
\vartheta_{n}(\xi)=1 \quad(n=1,2, \ldots)
$$

gelten.

Bisher ist (1), von dem bekannten Fall $n=1$ abgesehen, nur für $n=2$ bewiesen worden, nämlich von J. F. KubILyus [5]. Einer der Verfasser [4] gab kürzlich für diesen Satz einen vereinfachten Beweis und erzielte zugleich das bestmögliche Ergebnis für den komplexen Fall, wonach für fast alle komplexen $\xi$

$$
\vartheta_{2}(\xi)=\frac{1}{4}
$$

gilt. Für allgemeines $n$ dagegen besagt die bisher beste Vorstufe der Mahlerschen Vermutung, ein Satz von W. J. Leveque [6], nur, daß für fast alle reellen bzw. fast alle komplexen $\xi$ die Ungleichung

$$
1 \leqq \vartheta(\xi) \leqq 2, \text { bzw } \cdot \frac{1}{2} \leqq \vartheta(\xi) \leqq \frac{3}{2}
$$

gilt. Hauptziel der vorliegenden Arbeit ist der folgende

$\left.{ }^{1}\right)$ Siehe TH. Schneider [8], 3. Kap. 
Satz 1. a) Für fast alle reellen $\xi$ ist

$$
1 \leqq \vartheta_{n}(\xi) \leqq 2-\frac{2}{n} \quad(n=3,4, \ldots) .
$$

b) Für fast alle komplexen $\xi$ ist

$$
\frac{1}{2}-\frac{1}{2 n} \leqq \vartheta_{n}(\xi) \leqq \frac{3}{2}-\frac{2}{n} \quad(n=3,4, \ldots)
$$

Aussage a) ist offenbar für $n=2$ mit dem Resultat von J. F. KuBILyus identisch; dieses wird jedoch hier nicht bewiesen, da die hier angewandte Methode von der in [5] und auch von der in [4] abweicht und nicht auf den Fall $n=2$ übertragbar ist. Aussage b) würde sich mit unserer Methode zwar auch für den Fall $n=2$ beweisen lassen, jedoch dann weniger scharf sein als (2). Satz 1, der offensichtlich das Ergebnis von W. J. Leveque einschließt, wird sodann durch Einführung des Hausdorffschen Dimensionsbegriffs verschärft (Satz 2). Man erhält speziell die Aussage, daß die Menge aller T-Zahlen und die Menge aller $U$-Zahlen im Sinne der Mahlerschen Klasseneinteilung beide die Hausdorffsche Dimension Null haben.

2. Zum Beweis von Satz 1 benötigen wir die folgenden Hilfssätze. Die darin auftretenden Größen $C_{1}, C_{2}, \ldots$ bedeuten von $H$ unabhängige Konstanten, die jedoch von $n$ abhängen dürfen.

Hilfssatz 1. Für jede S-Zahl $\xi$ und jedes natïrliche $n$ ist $\vartheta_{n}(\xi) \geqq 1$ bzw. $v_{n}(\xi) \geqq \frac{1}{2}-\frac{1}{2 n}$, je nachdem, ob $\xi$ reell oder komplex $i$ st.

Beweis. Siehe Th. Schnemder [8], Seite 69.

Hilfssatz 2. Sei $P(x)=a_{0} x^{n}+\cdots+a_{n} \in \mathfrak{P}(n, H)$ ein Polynom mit den Vullstellen $\alpha_{1}, \ldots, \alpha_{n}$. Dann gilt, wenn die Indizes $i_{1}, \ldots, i_{m}$ voneinander verschieden sind,

$$
\left|\alpha_{i_{1}} \alpha_{i_{2}} \ldots \alpha_{i_{m}}\right| \leqq C_{1} \frac{H}{\left|a_{0}\right|} .
$$

Beweis ${ }^{2}$ ). Die Nullstellen von $P(x)$ seien dem absoluten Betrag nach geordnet:

$\alpha_{1}|\leqq| \alpha_{2}|\leqq \cdots \leqq| \alpha_{n_{1}}\left|\leqq \frac{1}{2}<\right| \alpha_{n_{1}+1}|\leqq \cdots \leqq| \alpha_{n_{2}}|\leqq 1<| \alpha_{n_{2}+1}|\leqq \cdots \leqq| \alpha_{n} \mid$.

Für jedes $x=z$ mit $|z|=1$ gilt offenbar

Daraus folgt

$$
|P(z)| \leqq(n+1) H .
$$

$$
\prod_{i=n_{1}+1}^{n}\left|z-\alpha_{i}\right|=\left|\frac{P(z)}{a_{0}} \prod_{i=1}^{n_{1}} \frac{1}{\left|z-\alpha_{i}\right|}\right| \leqq(n+1) 2^{n_{1}} \frac{H}{\left|a_{0}\right|} .
$$

Auf Grund des Satzes vom Maximum einer analytischen Funktion erhält man damit

$$
\prod_{i=n_{2}+1}^{n}\left|\alpha_{i}\right| \leqq 2^{n_{2}-n_{1}} \prod_{i=n_{1}+1}^{n}\left|\alpha_{i}\right| \leqq(n+1) 2^{n_{2}}-\frac{H}{\left|a_{0}\right|},
$$

woraus die Behauptung unmittelbar folgt.

$\left.{ }^{2}\right)$ Siehe N. I. Feldman [2]; der Beweis wird der Vollständigkeit halber hier angegeben. 
Hilfssatz 3 . Sei $n \geqq 2$ und $P(x)$ ein Polynom aus $\mathfrak{P}(n, H)$ mit einer von Null verschiedenen Diskriminante $D(P)$. Dann gilt für jede seiner Nullstellen $\alpha_{m}$ :

Beweis. Offenbar ist

$$
\left|P^{\prime}\left(\alpha_{m}\right)\right| \geqq C_{2} \frac{\sqrt{|D(P)|}}{H^{n-2}} .
$$

$$
|D(P)|=\left|a_{0}^{2 n-2} \prod_{i<j}\left(\alpha_{i}-\alpha_{j}\right)^{2}\right|=\left|a_{\substack{j=1 \\ j \neq m}}^{n}\left(\alpha_{m}-\alpha_{j}\right)^{2}\right| \cdot\left|\alpha_{0}^{2 n-4} \prod_{\substack{i<j \\ i, j \neq m}}\left(\alpha_{i}-\alpha_{j}\right)^{2}\right|,
$$

und wegen

folgt somit

$$
\left|a_{0}^{2} \prod_{\substack{j=1 \\ j \neq m}}^{n}\left(\alpha_{m}-\alpha_{j}\right)^{2}\right|=\left|P^{\prime}\left(\alpha_{m}\right)\right|^{2}
$$

$$
\left|P^{\prime}\left(\alpha_{m}\right)\right|=\frac{V|D(P)|}{\left|a_{0}\right|^{n-2}\left|\prod_{\substack{i<j \\ i, j \neq m}} \prod_{i}^{-1}\left(\alpha_{i}-\alpha_{j}\right)\right|} .
$$

Bei Ausmultiplikationen des Produktes im Nenner entsteht eine Summe von $2^{\left(\begin{array}{c}n-1 \\ 2\end{array}\right)}$ Summanden, von denen jeder seinerseits ein Produkt von $\left(\begin{array}{c}n-1 \\ 2\end{array}\right)$ Faktoren aus der Menge der $n-1$ Wurzeln $\alpha_{i}$ mit $i \neq m$ ist. In diesen Produkten kommt jedes $\alpha_{i}$ höchstens in der $(n-2)$-ten Potenz vor. Daher gilt nach Hilfssatz 2

$$
\left|a_{0}^{n-2} \prod_{\substack{i<j \\
i, j \neq m}}\left(\alpha_{i}-\alpha_{j}\right)\right| \leqq\left|a_{0}\right|^{n-2} 2^{\left(\begin{array}{c}
n-1 \\
2
\end{array}\right)}(n+1)^{n-2} 2^{n(n-2)} \frac{H^{n-2}}{\left|a_{0}\right|^{n-2}}=C_{3} H^{n-2},
$$

so daß sich die Behauptung nach (4) ergibt.

Hilfssatz 4. Unter den Voraussetzungen von Hilfssatz 3 gilt, wenn $\xi$ eine beliebige reelle oder komplexe $Z$ ahl und

ist,

$$
\left|\xi-\alpha_{m}\right|=\min _{i=1, \ldots, n}\left|\xi-\alpha_{i}\right|
$$

$$
\left|\xi-\alpha_{m}\right| \leqq C_{4} \frac{H^{n-2}}{\sqrt{|\overline{D(P)}|}}|P(\xi)| .
$$

Beweis. Da $\left|\alpha_{m}-\alpha_{i}\right| \leqq\left|\alpha_{m}-\xi\right|+\left|\xi-\alpha_{i}\right| \leqq 2\left|\xi-\alpha_{i}\right|$ ist, folgt

Somit ist nach Hilfssatz 3

$$
\left|P^{\prime}\left(\alpha_{m}\right)\right| \leqq 2^{n-1}\left|a_{0}\right| \prod_{\substack{i=1 \\ i \neq m}}^{n}\left|\xi-\alpha_{i}\right|
$$

$$
1 \leqq \frac{1}{C_{2}} 2^{n-1} \frac{H^{n-2}}{\sqrt{|| D(P) \mid}}\left|a_{0}\right| \prod_{\substack{i=1 \\ i \neq m}}^{n}\left|\xi-\alpha_{i}\right|,
$$

und hieraus folgt die Behauptung durch Multiplikation mit $\left|\xi-\alpha_{m}\right|$.

Hilfssatz 5. Seien $\tilde{w}_{n}(H, \xi), \tilde{w}_{n}(\xi), \widetilde{\vartheta}_{n}(\xi)$ und $\widetilde{\vartheta}(\xi)$ analog den Ausdrücken $w_{n}(H, \xi), w_{n}(\xi), \vartheta_{n}(\xi)$ und $\vartheta(\xi)$ definiert, jedoch unter Beschränkung auf irre. duzible Polynome $P(x)$. Dann gibt es für jedes $\xi$ und jedes n eine natüliche Zahl 
$v=v(n) \leqq n$ so, $d a \beta$

$$
\widetilde{\vartheta}_{n}(\xi) \leqq \vartheta_{n}(\xi) \leqq \widetilde{\vartheta}_{v}(\xi)
$$

gilt.

Beweis. Der linke Teil von (5) folgt unmittelbar aus der Tatsache, daß die Menge aller irreduziblen Polynome von einem Grad $\leqq n$ und einer Höhe $\leqq H$ eine Untermenge von $\mathfrak{P}(n, H)$ ist $\left.{ }^{3}\right)$. Zum Beweis des rechten Teils betrachten wir ein Polynom $P(x) \in \mathfrak{P}(n, H)$ mit $|P(\xi)|=w_{n}(H, \xi)$. Es sei $P=P_{1} \ldots P_{r}$ mit irreduziblen Polynomen $P_{\varrho}$; ferner sei Grad $P_{\varrho}=n_{\varrho}$ und Höhe $P_{\varrho}=H_{\varrho}$. Dann ist wegen $|P(\xi)|=\prod_{\varrho=1}^{r}\left|P_{\varrho}(\xi)\right|$ und

$$
\left|P_{\varrho}(\xi)\right| \geqq \widetilde{w}_{n_{\varrho}}\left(H_{\varrho}, \xi\right) \text { offenbar } u_{n}(H, \xi) \geqq \underset{\prod_{\varrho=1}^{r}}{\tilde{w}_{n_{\varrho}}}\left(H_{\varrho}, \xi\right),
$$

also

$$
\frac{\log \frac{1}{w_{n}(H, \xi)}}{\log H} \leqq \sum_{\varrho=1}^{r} \frac{\log \frac{1}{\widetilde{w}_{n_{0}}\left(H_{0}, \xi\right)}}{\log H} \leqq \sum_{\varrho=1}^{r}\left(1+\frac{\log c_{n}}{\log H}\right) \frac{\log \frac{1}{\widetilde{w}_{n_{\varrho}}\left(c_{n} H, \xi\right)}}{\log c_{n} H}
$$

sobei in der zweiten Ungleichung benutzt worden ist, daß es eine nur von $n$ Łbhängende Konstante $c_{n}$ mit $H_{\varrho} \leqq c_{n} H$ gibt $^{4}$ ). Durch indirekte Schlußweise colgt daraus ${ }^{5}$ )

Iso

$$
w_{n}(\xi) \leqq \max _{n_{1}+n_{3}+\cdots+n_{r}=n} \sum_{\varrho=1}^{\stackrel{r}{r}} \tilde{w}_{n_{\varrho}}(\xi)
$$

$$
\begin{aligned}
\vartheta_{n}(\xi) & \leqq \max _{n_{1}+n_{2}+\cdots+n_{r}=n} \frac{\widetilde{w}_{n_{1}}(\xi)+\cdots+\widetilde{w}_{n_{r}}(\xi)}{n_{1}+\cdots+n_{r}} \\
& \leqq \max _{n_{1}+n_{2}+\cdots+n_{r}=n}\left(\frac{\widetilde{w}_{n_{1}}(\xi)}{n_{1}}, \ldots, \frac{\widetilde{w}_{n_{r}}(\xi)}{n_{r}}\right),
\end{aligned}
$$

d. h. es ist $\vartheta_{n}(\xi) \leqq \tilde{\vartheta}_{v}(\xi)$ für ein gewisses $v \leqq n$, wie behauptet.

Ist also für eine Zahl $\xi$ eine Abschätzung der Form

$$
\tilde{\vartheta}_{v}(\xi) \leqq F(v) \quad(v=1,2, \ldots)
$$

bewiesen, bei der $F(v)$ cine monoton wachsende Funktion ist, so gilt für jedes natürliche $n$ auch

$$
\vartheta_{n}(\xi) \leqq \widetilde{\vartheta}_{\nu(n)}(\xi) \leqq F(\nu(n)) \leqq F(n)
$$

Daher werden wir uns beim Beweis von Satz 1 auf irreduzible Polynome beschränken können; doch wird davon nur in Form der Voraussetzung Gebrauch gemachi, daß keine mehrfachen Nullstelien auftrcten sollen.

3) Vgl. auch Abschn. 6 dieser Arbeit.

4) Siehe z. B. [8], 3. Kap., Hilfssatz 16.

5) Zu beachten ist, daß die Zerlegung $n_{1}+n_{2}+\cdots+n_{r}=n$ von $H$ abhängt. 
Der folgende Hilfssatz erscheint uns auch unabhängig von dem hier vorliegenden Zusammenhang bemerkenswert; er wird daher etwas allgemeiner formuliert, als es für das Folgende notwendig ist.

Hilfssatz 6. Zu jeder Zahl $\beta>0$ und jeder natürlichen Zahl $n \geqq 2$ gibt es eine Konstante $C>0$ derart, da $\beta$ für jedes Polynom $V(x)$ vom Grad $n$ mit ganzrationalen Koeffizienten und alle natïrlichen Zahlen $H$ gilt:

$$
\sum_{\substack{x=-H \\ V(x) \neq 0}}^{H} \frac{1}{|V(x)|^{\beta}}< \begin{cases}C H^{1-\beta n} & \text { für } \beta n<1, \\ C \log (H+1) & \text { für } \beta n=1, \\ C & \text { für } \beta n>1\end{cases}
$$

Beweis. Das Intervall $[-H, H]$ werde durch die Punkte

$$
x_{0}=-H, x_{1}, \ldots, x_{q}=H
$$

so in Teilintervalle $\left[x_{i}, x_{i+1}\right]$ zerlegt, daß in jedem von ihnen die Polynome ${ }^{6}$ ) $V(x), \Delta V(x), \ldots, \Delta^{n-1} V(x)$ sämtlich monoton und im Innern von Null verschieden sind. Dies ist bei festem $V(x)$ offenbar mit einem $q \leqq n^{2}+1$ möglich. Dann genügt es, die Behauptung für ein einziges Intervall $\left[x_{i}, x_{i \div 1}\right]$ an Stelle von $[-H, H] \mathrm{zu}$ beweisen.

Auf Grund der Wahl der $x_{i}$ sind auch die Funktionen

$$
F_{j}(x)=\left|\Delta^{n-j} V(x)\right| \quad(j=1, \ldots, n)
$$

sämtlich in den Intervallen $\left[x_{i}, x_{i+1}\right]$ monoton. Daher gilt, wenn $x_{i} \leqq x \leqq x_{i+1}-1$ ist,

(6) $\Delta F_{j+1}(x)=\left\{\begin{array}{r}F_{j}(x), \text { falls } F_{j+1}(x) \text { in }\left[x_{i}, x_{i+1}\right] \text { monoton wächst, } \\ -F_{j}(x), \text { falls } F_{j+1}(x) \text { in }\left[x_{i}, x_{i+1}\right] \text { monoton fällt. }\end{array}\right.$

Sei $r_{i}$ die kleinste ganze Zahl $\geqq x_{i}$ und $s_{i}=\left[x_{i+1}\right]$. Im folgenden denken wir uns das Intervall $\left[x_{i}, x_{i+1}\right]$ festgehalten und betrachten nur ganze Zahlen $x \in\left[x_{i}, x_{i+1}\right]$. Durch Induktion soll gezeigt werden:

$$
F_{j}(x) \geqq \begin{cases}\frac{1}{j !}\left(x-r_{i}-(j-1)\right)^{j} & \text { für } r_{i}+j-1 \leqq x \leqq s_{i}, \\ \frac{1}{j !}\left(s_{i}-x-(j-1)\right)^{j} & \text { falls } F_{j}(x) \text { monoton wächst, } \\ & \text { für } r_{i} \leqq x \leqq s_{i}-(j-1) \\ & \text { falls } F_{j}(x) \text { monoton fällt. }\end{cases}
$$

Wir nehmen zuerst an, die Funktion $F_{1}(x)$ sei in $\left[x_{i}, x_{i+1}\right]$ monoton wachsend. Da sie auf Grund der Voraussetzungen linear und ganzwertig ist, folgt

$$
F_{1}\left(r_{i}\right) \geqq 0 \text { und } F_{1}(x) \geqq x-r_{i} \text { für } \quad r_{i} \leqq x \leqq s_{i} .
$$

Analog ergibt sich, falls $F_{1}(x)$ monoton fällt,

$$
F_{1}\left(s_{i}\right) \geqq 0 \text { und } F_{1}(x) \geqq s_{i}-x \text { für } r_{i} \leqq x \leqq s_{i} .
$$

Die Behauptung (7) sei nun für ein $j$ mit $1 \leqq j<n$ erfüllt. Ist $F_{j+1}(x)$ monoton

$\left.{ }^{6}\right)$ Dabei ist, wie üblich, $\Delta F(x)$ die Funktion $F(x+1)-F(x)$ und $\Delta^{j+1}=\Delta\left(\Delta^{j}\right)$. 
wachsend bzw. fallend, so gilt ${ }^{7}$ )

bzw.

$$
F_{j \div 1}(x)=F_{j+1}\left(r_{i}\right)+\sum_{v=r_{i}}^{x-1} \Delta F_{j \div 1}(v)
$$

$$
F_{j+1}(x)=F_{j+1}\left(s_{i}\right)-\sum_{v=x}^{s_{i}-1} \Delta F_{j+1}(v) .
$$

Wegen (6) und (7) erhält man daraus im ersten Falle ${ }^{7 a}$ ) für $r_{i}+j \leqq x \leqq s_{i}$ :

$$
\begin{aligned}
& F_{j+1}(x)=F_{j+1}\left(r_{i}\right)+\sum_{v=r_{i}}^{x-1} \Delta F_{j+1}(v) \geqq \sum_{r=r_{i}+j-1}^{x-1} F_{j}(v) \\
& \geqq \frac{1}{j !} \sum_{v=r_{i}+j-1}^{x-1}\left(v-r_{i}-(j-1)\right)^{j} \geqq \frac{1}{j !} \int_{0}^{x-r_{i}-j} t^{j} d t=\frac{1}{(j+1) !}\left(x-r_{i}-j\right)^{j+1}
\end{aligned}
$$

Analog folgt im zweiten Fall ${ }^{7 a}$ ) für $r_{i} \leqq x \leqq s_{i}-j$ :

$$
\begin{aligned}
& F_{j+1}(x)=F_{j+1}\left(s_{i}\right)-\sum_{v=x}^{s_{i}-1} \Delta F_{j+1}(v) \geqq \sum_{v=x+1}^{s_{i}-j+1} F_{j}(v) \\
& \geqq \frac{1}{j !} \sum_{v=\frac{1}{2}+1}^{s_{i}-j+1}\left(s_{i}-v-(j-1)\right)^{j} \geqq \frac{1}{j !} \int_{0}^{s_{i}-x-j} t^{j} d t=\frac{1}{(j+1) !}\left(s_{i}-x-j\right)^{j+1} .
\end{aligned}
$$

Damit ist (7) bewiesen, und man erhält speziell:

$$
F_{n}(x)=|V(x)| \geqq \begin{cases}\frac{1}{n !}\left(x-r_{i}-(n-1)\right)^{n} & \text { für } r_{i}+n-1 \leqq x \leqq s_{i}, \\ & \text { falls }|V(x)| \text { monoton wächst }, \\ \frac{1}{n !}\left(s_{i}-x-(n-1)\right)^{n} & \text { für } r_{i} \leqq x \leqq s_{i}-(n-1), \\ & \text { falls }|V(x)| \text { monoton fällt. }\end{cases}
$$

Aus diesen Ungleichungen folgt

$$
\sum_{x=r_{i}+n}^{s_{i}-n} \frac{1}{V(x)^{\beta}} \leqq(n !)^{\beta} \quad \sum_{x=1}^{s_{i}-r_{i}}{ }^{2 n+1} \frac{1}{x^{\beta} n} .
$$

Schätzt man noch $|V(x)|$ für die restlichen Werte $r_{i} \leqq x<r_{i}+n$ und $s_{i}-n<x \leqq s_{i}$ trivial durch $|V(x)| \geqq 1 \mathrm{ab}$, so folgt

$$
\begin{aligned}
& \sum_{\substack{x \equiv r_{i} \\
I^{\prime}(x) \neq 0}}^{s_{i}} \frac{1}{\mid V(x)^{\beta}} \leqq 2 n+(n !)^{\beta} \quad \sum_{x=1}^{s_{i}-r_{i}-2 n+1} \frac{1}{x^{\beta n}} \\
& \leqq 2 n+(n !)^{\beta} \quad \int_{1}^{s_{i}-r_{i}} t^{-\beta n} d t \leqq 2 n+(n !)^{\beta} \int_{1}^{2 H} t^{-\beta n} d i,
\end{aligned}
$$

7) Ist der obere Summationsindex einer Summe kleiner als der untere, so soll sie den Wert 0 haben.

`a) Siehe ,Zusatz bei der Korrektur“ am Ende der Arbeit. 
wobei die Ungleichung jetzt für alle Intervalle gilt. Wegen

folgt die Behauptung.

$$
\int_{1}^{2 H} t^{-\beta n} d t \leqq \begin{cases}\frac{2^{1-\beta}}{1-\beta n} H^{1-\beta n} & \text { für } \quad \beta n<1, \\ \log 2 H & \text { für } \quad \beta n=1 \\ \frac{1}{\beta n-1} & \text { für } \quad \beta n>1\end{cases}
$$

Hilfssatz 7. Ist $n \geqq 3$, so treten in dem Polynom

$$
D\left(a_{0}, a_{1}, \ldots, a_{n}\right)=\text { Diskriminante von } a_{0} x^{n}+a_{1} x^{n-1}+\cdots+a_{n}
$$

wenigstens zwei der $n+1$ Veränderlichen $a_{i}$ in höherer als erster Potenz auf.

Beweis. Aus der Resultantendarstellung der Diskriminante folgt unmittelbar, daß in ihr das Glied $n^{n} a_{0}^{n-1} a_{n}^{n-1}$ auftritt.

3. Wir beweisen nun Satz 1, wobei wir uns im Hinblick auf Hilfssatz 1 auf den rechten Teil beider Behauptungen beschränken können. Zum Beweis von a) betrachten wir bei festem $\sigma>0$ und natürlichem $n$ die Menge $\mathfrak{R}_{n}(\sigma)$ der reellen Zahlen $\xi$ mit $\vartheta_{n}(\xi)>1+\sigma$. Wie aus der Definition von $\vartheta_{n}(\xi)$ folgt, gehört eine Zahl $\xi$ genau dann zu $\mathfrak{R}_{n}(\sigma)$, wenn zu unendlich vielen $H$ ein Polynom $P$ aus der Komplementärmenge $\mathfrak{P}_{0}(n, H)$ von $\mathfrak{P}(n, H-1)$ in $\mathfrak{P}(n, H)$ mit

$$
0<|P(\xi)|<H^{-n(1+\sigma)}
$$

existiert. Wir ordnen jeder Nullstelle $\alpha_{i}$ eines Polynoms $P \in \mathcal{P}^{3}(n, H)$ das Intervall

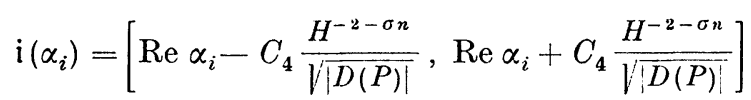

zu. Dann gilt für jedes reelle $\xi$ mit (8), wenn $\alpha_{i}$ die nächstliegende Nullstelle von $P(x)$ ist, nach Hilfssatz 4

$$
\left|\xi-\operatorname{Re} \alpha_{i}\right| \leqq\left|\xi-\alpha_{i}\right|<C_{4} \frac{H^{n-2}|P(\xi)|}{\sqrt{|D(P)|}}<C_{4} \frac{H^{-2-\sigma n}}{\sqrt{|D(P)|}},
$$

d. h. $\xi \in \mathfrak{i}\left(\alpha_{i}\right)$. Folglich wird $\mathfrak{R}_{n}(\sigma)$ für jedes $H_{0}$ durch die Gesamtheit dieser Intervalle $\mathrm{i}\left(\alpha_{i}\right)$ mit zugehörigen Polynomen $P \in \mathfrak{P}_{0}(n, H), H \geqq H_{0}$, überdeckt. Also ist, wenn man mit $\mu_{1}$ das lineare Lebesguesche Maß bezeichnet ${ }^{8}$ ),

$$
\mu_{1}\left(\Re_{n}(\sigma)\right) \leqq \lim _{H_{0} \rightarrow \infty} \sum_{H=H_{0}}^{\infty} \sum_{P \in \mathcal{P}_{0}(n, H)}^{\prime} C_{4} \frac{H^{-2-\sigma n}}{\mid \sqrt{|D(P)|}} .
$$

Zur Abschätzung der Summe

$$
\Sigma=\sum_{P \in \mathfrak{P}_{0}(n, H)} \frac{1}{\sqrt{|D(P)|}}
$$

haben wir $\mathrm{zu}$ berücksichtigen, daß wenigstens ein Koeffizient, etwa $a_{i}$, den Betrag $H$ hat. Hält man dann alle Koeffizienten bis auf einen, etwa $a_{j}$ mit $j \neq i$, fest, so wird $D(P)$ ein Polynom $V\left(a_{j}\right)$ in $a_{j}$, dessen Grad nach Hilfssatz 7 bei geeigneter Wahl von $j$ sicher $\geqq 2$ ist. Daher wird nach Hilfssatz 6

$\left.{ }^{8}\right)$ Der Strich am Summenzeichen soll andeuten, daß nur Polynome $P$ ohne mehrfache Nullstellen betrachtet werden, für die also $D(P) \neq 0$ ist. 
für $\varepsilon>0$ bei genügend großem $H$

$$
\Sigma<C_{8} H^{n-1} \sum_{\substack{a_{j}=-H \\ V\left(a_{j}\right) \neq 0}}^{H} \frac{1}{\sqrt{\left|V\left(a_{j}\right)\right|}}<C_{9} H^{n-1+\varepsilon} .
$$

Folglich ergibt sich auf Grund von (9)

$$
\mu_{1}\left(\Re_{n}(\sigma)\right) \leqq C_{10} \varliminf_{H_{0} \rightarrow \infty} \sum_{H=H_{0}}^{\infty} H^{-2-o n+n-1+\varepsilon} .
$$

Ist nun $\sigma>1-\frac{2-\varepsilon}{n}$, so wird in der letzten Reihe der Exponent $<-1$, so daß Konvergenz eintritt und $\mu_{1}\left(\mathfrak{R}_{n}(\sigma)\right)=0$ folgt. Läßt man $\sigma$ eine Folge von Werten $\sigma_{1}, \sigma_{2}, \ldots$ mit $\sigma_{i}>\sigma_{i+1}$ und $\lim _{i \rightarrow \infty} \sigma_{i}=1-\frac{2}{n}$ durchlaufen, so erhält man

und daher

$$
\bigcup_{i=1}^{\infty} \Re_{n}\left(\sigma_{i}\right)=\Re_{n}\left(1-\frac{2}{n}\right)
$$

$$
\mu_{1}\left(\bigcup_{i=1}^{\infty} \Re_{n}\left(\sigma_{i}\right)\right)=\mu_{1}\left(\Re_{n}\left(1-\frac{2}{n}\right)\right)=0
$$

Also hat die Menge aller reellen Zahlen $\xi$ mit $\vartheta_{n}(\xi) \leqq 2-\frac{2}{n}$, wie behauptet, das Lebesguesche Maß Eins.

Der Beweis der Aussage b) verläuft analog. Man betrachtet für festes $\sigma>0$ die Menge $\mathfrak{R}_{n}(\sigma)$ der komplexen Zahlen $\xi$ mit $\vartheta_{n}(\xi)>\frac{1}{2}+\sigma$. An Stelle des Intervalls $\mathfrak{i}\left(\alpha_{i}\right)$ verwenden wir jeweils den Kreis $\mathfrak{f}\left(\alpha_{i}\right)$ der Zahlen $z$ mit $\left|z-\alpha_{i}\right| \leqq C_{4} \frac{H^{n\left(\frac{1}{2}-\sigma\right)-2}}{\sqrt{|D(P)|}}$. Dann crkennt man wie oben, daß jedes $\xi \in \Re_{n}(\sigma)$ von einem solchen Kreis überdeckt wird. Statt (10) erhält man, wenn man die Summe $\sum_{1 \in \vartheta_{i}(n, H)}^{\Gamma^{\prime}} \frac{1}{|D(P)|}$ mit Hilfssatz 6 abschätzt und mit $\mu_{2}$ das zweidimen. sionale Lebeguesche Maß bezeichnet, die Ungleichung $\mu_{2}\left(\Re_{n}(\sigma)\right) \leqq \lim _{H_{0} \rightarrow \infty} \sum_{I I=H_{0}}^{\infty} I_{I^{\prime} \in \Re_{0}(n, H)}^{\sum^{\prime}} C_{11} \frac{H^{n(1-2 \sigma)-4}}{|D(P)|} \leqq C_{12}{\underset{H}{H_{0} \rightarrow \infty}}_{H=H_{0}} \sum^{\infty} H^{n(1-2 \sigma)+n-5}$.

Daraus ergibt sich ähnlich wie oben $\mu_{2}\left(\Omega_{n}\left(1-\frac{2}{n}\right)\right)=0$, womit alles bewiesen ist.

4. Die Aussagen von Satz 1 lassen sich verallgemeinern, wenn man statt des Lebesgueschen Maßes den Hausdorffschen Maß- und Dimensionsbegriff ${ }^{9}$ ) heranzieht. Man erhält so den

Satz 2. Fïr jedes $\sigma \geqq 0$ und $n \geqq 3$ ist

$$
\operatorname{dim} \Re_{n}(\sigma) \leqq \frac{n+1}{\sigma n+3}
$$

$\left.{ }^{9}\right)$ Definitionen und hier verwendete Schreibweise findet man z. B. bei B. VoLKMANN [9]. 
und

$$
\operatorname{dim} \Re_{n}(\sigma) \leqq\left\{\begin{array}{l}
\frac{n}{\left(\sigma-\frac{1}{2}\right) n+2}, \text { falls } \frac{1}{2}-\frac{2}{n} \leqq \sigma \leqq \frac{3}{2}-\frac{2}{n}, \\
\frac{n+1}{\left(\sigma-\frac{1}{2}\right) n+3}, \text { falls } \frac{3}{2}-\frac{2}{n} \leqq \sigma .
\end{array}\right.
$$

Es sei bemerkt, daß man, um aus diesem Satz nichttriviale Abschätzungen, also solche von der Form $\operatorname{dim} \Re_{n}(\sigma)<1$ bzw. $\operatorname{dim} \Re_{n}(\sigma)<2$ zu erhaltev, dic Ungleichungen (11) und (12) auf den Bereich $\sigma>1-\frac{2}{n}$ beschränken muß, wie dies gemäß Satz 1 zu erwarten ist.

Beweis. a) Für jedes $H_{0}$ bildet die Menge der Intervalle $\mathrm{i}\left(\alpha_{i}\right)$ mit zugehörigem $H \geqq H_{0}$ eine $\left(2 C_{4} H_{0}^{-2-\sigma n}\right)$-Überdeckung von $\Re_{n}(\sigma)$. Daher gil bei gegebenem $\delta$ mit $0<\delta<1$ für das $\delta$-dimensionale Hausdorffsche Maß

$$
\left\{\Re_{n}(\sigma)\right\}^{\delta} \leqq \lim _{H_{0} \rightarrow \infty} C_{10} \sum_{H=H_{0} I^{\prime} \in \mathcal{Y}_{0}(n, H)}^{\infty} \sum^{\prime \prime}\left(\frac{H^{-2-\sigma n}}{\sqrt{|(P)|}}\right)^{\delta} .
$$

Für die Summe $\sum_{P \in \Re_{0}(n . H)}^{\prime}|D(P)|^{-\delta / 2}=\Sigma$ erhält man nach Hilfssatz 6 die Abschätzung $\Sigma \leqq C_{11} H^{1-\delta \div n-1}=C_{11} H^{n-\delta}$, so daß sich schließlich

$$
\left\{\mathcal{R}_{n}(\sigma)\right\}^{\delta} \leqq \lim _{H_{0} \rightarrow \infty} C_{11} \sum_{H=H_{0}}^{\infty} I t^{-\delta(\sigma n+3)+n}
$$

ergibt. Somit gilt $\left\{\bigcap_{n}(\sigma)\right\}^{\delta}=0$ für alle $\delta>\frac{n+1}{\sigma n+3}$, woraus der erste Teil der Behauptung folgt.

b) Der Beweis für den komplexen Fall verläuft analog; nur muß man Werte $\delta$ mit $0<\delta<2$ zulassen, so daß sich - unter Verwendung ron Hilfssatz 6 - die Abschätzung

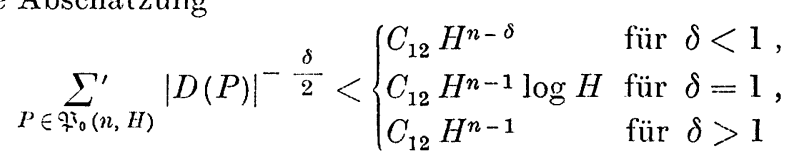

ergibt, die dann zur Unterscheidung der beiden in der Behauptung (12) auftretenden Fälle führt.

5. Der Vollständigkeit halber führen wir auch Dimensionsabschätzungen für die beiden in Satz 3 nicht erwähnten Fälle $n=1$ und $n=2$ an. Für $n=1$ ist nach $\mathrm{v}$. JARNík [3]

$$
\operatorname{dim} \Re_{1}(\sigma)=\frac{2}{\sigma+1}(\sigma \geqq 1) .
$$

Ferner bestätigt man leicht, daß

$$
\mathfrak{R}_{1}(\sigma)=\mathfrak{R}_{1}\left(\sigma-\frac{1}{2}\right)
$$

gilt.

Im Fall $n=2$ gilt der

Hilfssatz 8. Zu jedem $\beta$ mit $0<\beta<1$ gibt es von $H$ unabhängige positive Zahlen $c$ und $\tau$ so, daß gilt:

$$
\sum_{P \in \mathfrak{P}_{0}(2, H)}^{\prime}|D(P)|^{-\beta}<c H^{\frac{\tau}{\log \log H}+2(1-\beta)} .
$$


Beweis. Wir übernehmen aus [4] die Abschätzung ${ }^{10}$ )

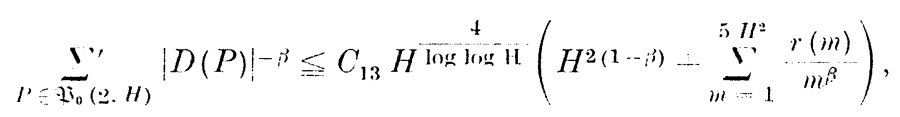

wobei $r^{2}(m)$ der größte gemeinsame quadratische Teiler von $m$ und $4 H$ ist. In der rechts stehenden Summe kommen zu vorgegebenem $r(m)=k$ höchstens die folgenden Glieder vor:

$$
\left[\begin{array}{c}
5 H^{2} \\
k \\
\sum_{i}^{k}
\end{array} \frac{k}{(k i)^{\beta}} \leqq \frac{1}{1-\beta} k^{1-\beta}\left(\begin{array}{c}
5 H^{2} \\
k
\end{array}\right)^{1-i} \ldots C_{14} H^{2(1-\beta)} .\right.
$$

Die Anzahl der möglichen $k$ ist jedenfalls kleiner oder gleich der Anzahl $d(4 H)$ der Teiler von $4 H$, und dafür gilt bekanntlich

$$
d(4 H) \leqq C_{15} H^{\frac{2}{\log \log H}} .
$$

Die vorstehenden Ungleichungen zusammen liefern die Behauptung.

Auf Grund von Hilfssatz 8 erhält man, ähnlich wie beim Beweis von Satz 2 , die Abschätzungen

und

$$
\operatorname{dim} \Re_{2}(\sigma) \leqq \frac{3}{2 \sigma+3} \quad(\sigma \geqq 0)
$$

$$
\operatorname{dim} \mathfrak{R}_{2}(\sigma) \leqq \frac{2}{2 \sigma+2} \quad\left(\sigma \geqq-\frac{1}{4}\right)
$$

Aus Satz 2 und den obigen Betrachtungen ergeben sich zwei Folgerungen.

Folgerung 1. Bezeichnet man mit $\mathfrak{R}(\sigma)$ bzw. $R(\sigma)$ dic Menge der reellen bzw. komplexen $\xi$ mit $\vartheta(\xi)>1+\sigma$ bzw. $\vartheta(\xi)>\frac{1}{2}+\sigma$, so ist für $\sigma \geq 1$

und

$$
\operatorname{dim} \Re(\sigma)=\frac{2}{\sigma+1}
$$

$$
\operatorname{dim} \mathfrak{R}(\sigma) \leqq \stackrel{2}{\sigma} \stackrel{2}{2} \cdot \frac{1}{2} .
$$

Beweis. Ist $\xi \in \Re(\sigma)$, so gibt es ein $n$ mit $\vartheta_{n}(\xi)>1 \div \sigma$. Somit ist

und folglich

$$
\Re(\sigma) \subseteq \bigcup_{n=1}^{\infty} \Re_{n}(\sigma)
$$

also nach (13), \{15) und (11)

$$
\lim \Re(\sigma) \leqq \sup _{n=1}^{\infty} \operatorname{dim} \Re_{n}(\sigma)=\tau,
$$

$$
\tau \leqq \max \left(\frac{2}{\sigma+1}, \frac{3}{2 \sigma+3}, \sup _{n=3,4, \ldots} \frac{n+1}{\sigma n+3}\right)=\frac{2}{\sigma+1} .
$$

10) [4], Seite 267, Formeln (12) und (14).

Math. Ann. 136 
Ähnlich erhält man nach (14), (16), (12) und (11) die Abschätzungen

$$
\operatorname{dim} \mathfrak{R}(\sigma) \leqq\left\{\begin{array}{l}
\max \left(\frac{2}{\sigma+\frac{1}{2}}, \frac{3}{2 \sigma+2}, \sup _{n=3,4, \cdots} \frac{n}{\left(\sigma-\frac{1}{2}\right) n+2}\right) \text { für } 1 \leqq \sigma-\begin{array}{l}
3 \\
2
\end{array} . \\
\max \left(\frac{2}{\sigma+\frac{1}{2}}, \frac{3}{2 \sigma+2}, \sup _{n=3,4, \ldots} \frac{n+1}{\left(\sigma-\frac{1}{2}\right) n+3}\right) \text { für } \frac{3}{2} \leqq \sigma .
\end{array}\right.
$$

und daraus ergibt sich die zweite obige Behauptung.

Folgerung 2. Für die Mengen $T$ und $U$ aller $T$ - bzw. $U$-Zahlen ${ }^{11}$ ) gilt

$$
\operatorname{dim} T=\operatorname{dim} U=0 .
$$

Beweis. Für jedes (noch so große) $\sigma$ gilt $T \leqq \Omega(\sigma)$ und $U \subseteq \Re(\sigma)$.

6. Die Definition des Typs $\vartheta$ einer transzendenten Zahl $\xi$ läßt sich folgendermaßen verallgemeinern: Sei $\mathcal{Q}$ eine beliebige Menge von Polynomen mit ganzrationalen Koeffizienten und $\mathcal{Q}(n, H)$ die Menge der $Q \in \mathcal{Q} \operatorname{mit}$ Grad $Q \leqq n$ und Höhe $Q \leqq H$. Dann setzt man für jede transzendente Zahl $\xi$

$$
w_{n}(H, \xi, \mathfrak{Q})=\min _{Q \in \mathcal{Q}(n, H)}|Q(\xi)|
$$

und definiert $w_{n}(\xi, \mathfrak{Q}), \vartheta_{n}(\xi, \mathfrak{Q})$ und $\vartheta(\xi, \mathfrak{Q})$ entsprechend den Größen $w_{n}(\xi)$, $\vartheta_{n}(\xi)$ und $\vartheta(\xi)$. Für zwei solche Mengen $\mathfrak{Q}_{1}$ und $\mathfrak{Q}_{2}$ mit $\mathfrak{Q}_{1} \leqq \mathfrak{Q}_{2}$ gelten dann trivialerweise die Ungleichungen

$$
\begin{array}{rlrl}
w_{n}\left(H, \xi, \mathfrak{Q}_{1}\right) & \geqq w_{n}\left(H, \xi, \mathfrak{Q}_{2}\right), & \vartheta_{n}\left(\xi, \mathfrak{Q}_{1}\right) \leqq \vartheta_{n}\left(\xi, \mathfrak{Q}_{2}\right) \\
w_{n}\left(\xi, \mathfrak{Q}_{1}\right) \leqq w_{n}\left(\xi, \mathfrak{Q}_{2}\right), & \vartheta\left(\xi, \mathfrak{Q}_{1}\right) \leqq \vartheta\left(\xi, \mathfrak{Q}_{2}\right)
\end{array}
$$

für jedes $\xi$ und jedes natürliche $n$. Man kann nun die Frage aufwerfen, unter welchen Voraussetzungen über $\mathfrak{Q}$ das Analogon der Mahlerschen Vermutung bewiesen werden kann. Wir geben dazu den folgenden Satz an, dessen Beweis wie der von Satz 1 verläuft.

Satz 3. Sei $\mathfrak{Q}$ eine Menge von Polynomen mit ganzrationalen Koeffizienten, die mit jedem Polynom $Q$ auch alle seine Teiler als Elemente enthält, und $\tilde{\mathfrak{Q}}$ die Tcilmenge der Elemente von $\mathfrak{Q}$ ohne mehrfache Nullstellen. Gilt dann für ein natürliches $n$ beim Grenzübergang $H \rightarrow \infty$ die Aussage

$$
\underset{Q \in \widetilde{\Omega_{0}}(n, H)}{\sum^{\prime}} \frac{1}{\sqrt{D(Q)}}=O\left(H^{\sigma n}\right) \quad(\sigma>0, \text { fest })
$$

oder

$$
{\stackrel{\nu}{Q \in \widetilde{\Omega}_{0}(n, H)}}^{\prime} \frac{1}{|D(Q)|}=O\left(H^{\sigma n}\right)
$$

so ist für fast alle reellen $\xi$ die Ungleichung $\vartheta_{n}(\xi, Q) \leqq 1+\sigma-\frac{1}{n} b z w$. für fast alle komplexen $\xi$ die Ungleichung $\vartheta_{n}(\xi, Q) \leqq \frac{1+\sigma}{2}-\frac{3}{2 n}$ erfüllt.

Folgerung. Gilt (17) oder (18) für alle natürlichen $n$ (oder auch nur für unendlich viele), so gilt für fast alle reellen $\xi$ die Ungleichung $\vartheta(\xi, \mathfrak{Q}) \leqq 1+\sigma$ bzw. für fast alle komplexen $\xi$ die Ungleichung $\vartheta(\xi, \mathcal{Q}) \leqq \frac{1+\sigma}{2}$.

11) Siehe Th. Schnerder, a. a. O.1) 
Für Satz 3 lassen sich leicht Beispiele bilden, wenn man bedenkt, daß die Voraussetzungen (17) und (18) immer erfüllt sind, wenn die Anzahl $A_{n}(H)$ der Elemente von $\mathfrak{Q}_{0}(n, H)$ der Bedingung $A_{n}(H)=O\left(H^{\sigma n}\right)$ genügt. Dies trifft insbesondere dann zu, wenn man verlangt, daß die auftretenden Grade $n$ oder auch die Koeffizienten $a_{i}$ gewissen Mengen angehören sollen, über die man geeignete Dichtevoraussetzungen macht.

Zusatz bei der Korrektur. Im Beweis von Hilfssatz 6 sind nur die Fälle behandelt, da $\beta F_{j-1}(x)$ und $F_{j}(x)$ im Intervall $\left[x_{i}, x_{i \ldots 1}\right]$ entweder beide monoton wachsen oder beide monoton fallen. Nimmt man nun an, daß $F_{j, 1}(x)$ monoton wächst, aber $F_{j}(x)$ monoton fällt, so erhält man

$$
\begin{aligned}
F_{j+1}(x) \geqq \sum_{v=r_{i}+j-1}^{x-1} F_{j}(v) & \geqq \frac{1}{j !} \sum_{r=r_{i}+j-1}^{r-1}\left(s_{i}-v-(j-1)\right)^{j} \\
& \geqq \frac{1}{j !} \sum_{r=1}^{x-r_{i}-j+1} v^{j} \geqq \frac{1}{j !} \int_{0}^{x-r_{i} j} t^{j} d t,
\end{aligned}
$$

womit auch in diesem Falle der Induktionsschluß durchgeführt ist. Analog schließt man, wenn $F_{j-1}(x)$ monoton fällt, aber $F_{j}(x)$ monoton wächst.

Schließlich möchten wir erwähnen, daß Hilfssatz 6 einfacher und kürzer dadurch zu beweisen ist, da $\beta$ man von der Intervalleinteilung $-H, \beta_{1}, \ldots, \beta_{n}, H$ ausgeht, wobei $\beta_{1}, \ldots, \beta_{n}$ die nach wachsender Größe geordneten Realteile der Nullstellen von $V(x)$ sind, und die Summation über $|V(x)|^{-\beta}$ in diesen Intervallen einzeln ausführt.

\section{Literatur}

[1] Chintschin, A. J.: Zwei Bemerkungen zu einer Arbeit von Herm Perron. Math. Z. 22, 274-284 (1925). - - [2] Feldmax, N. I.: Approximation einiger transzendenter Zahlen. I. (russ.). Iswestija Akad. Nauk SSSR, Ser. mat. 15, 53-74 (1951). [3] JARNík, V.: Diophantische Approximationen und Hausdorffsches Maß. Mat. Sbornik 36, 371-382 (1929). - [4] KASCH, F.: Über eine metrische Eigenschaft der S-Zahlen. Math. Z. 70, 263-270 (1958). - [5] Kubilyus, J. F.: Über die Anwendung einer Winogradowschen Methode auf die Lösung eines Problems aus der metrischen Zahlentheorie (russ.). Dokl. Akad. Nauk SSSR, N.S. 67, 783-786 (1949). - [6] LEveque, W. J.: Note on S-numbers. Proc. Amer. Math. Soc. 4, 189-190 (1950). [7] Mahler, K.: Über das Maß der Menge aller S-Zahlen. Math. Ann. 106, 131-139 (1932). - [8] Schnerner, Th.: Einführung in die transzendenten Zahlen. BerlinGöttingen-Heidelberg: Springer 1957. - - [9] VoLkusx, B.: Über Hausdorffsche Dimensionen von Mengen, die durch Zifferneigenschaften charakterisiert sind. I. Math. \%. 58, 284-287 (1953).

(Eingegangen am 20. Juni 1958) 